\title{
Optical mapping of optogenetically shaped cardiac action potentials
}

SUBJECT AREAS:

ARRHYTHMIAS

ION CHANNELS IN THE NERVOUS SYSTEM

OPTOGENETICS

Received

19 March 2014

Accepted

30 July 2014

Published

19 August 2014

Correspondence and requests for materials should be addressed to

D.T.Y. (dyue@jhmi. edu)

\author{
Sarah A. Park' , Shin-Rong Lee ${ }^{2}$, Leslie Tung ${ }^{2}$ \& David T. Yue ${ }^{1,2,3,4}$
}

'Program in Cellular and Molecular Medicine, The Johns Hopkins University School of Medicine, Baltimore, MD, USA, ${ }^{2}$ Department of Biomedical Engineering, The Johns Hopkins University School of Medicine, Baltimore, MD, USA, ${ }^{3}$ Department of Neuroscience, The Johns Hopkins University School of Medicine, Baltimore, MD, USA, ${ }^{4}$ Center for Cell Dynamics, The Johns Hopkins University School of Medicine, Baltimore, MD, USA.

Light-mediated silencing and stimulation of cardiac excitability, an important complement to electrical stimulation, promises important discoveries and therapies. To date, cardiac optogenetics has been studied with patch-clamp, multielectrode arrays, video microscopy, and an all-optical system measuring calcium transients. The future lies in achieving simultaneous optical acquisition of excitability signals and optogenetic control, both with high spatio-temporal resolution. Here, we make progress by combining optical mapping of action potentials with concurrent activation of channelrhodopsin-2 (ChR2) or halorhodopsin (eNpHR3.0), via an all-optical system applied to monolayers of neonatal rat ventricular myocytes (NRVM). Additionally, we explore the capability of ChR2 and eNpHR3.0 to shape action-potential waveforms, potentially aiding the study of short/long QT syndromes that result from abnormal changes in action potential duration (APD). These results show the promise of an all-optical system to acquire action potentials with precise temporal optogenetics control, achieving a long-sought flexibility beyond the means of conventional electrical stimulation.

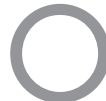

ptogenetics has revolutionized neuroscience ${ }^{1-4}$, offering exquisite control of cell excitability with a resolution and flexibility complementary to more traditional strategies involving electrical stimulation. In particular, optogenetics affords precise spatiotemporal control via light-activated unipolar injection of depolarizing ${ }^{1,5}$ (ChR2) and hyperpolarizing currents ${ }^{2,6}$ (eNpHR3.0), kindling strong interest in applications beyond neurons, such as in the heart. Hoffmann et al. ${ }^{7}$ demonstrated light-induced stimulation of confluent layers of HL- 1 cells expressing ChR2, recording electrical propagation with a microelectrode array. Bruegmann et al. ${ }^{8}$ undertook light-induced stimulation of ChR2-expressing murine muscle and heart. Arrenberg et al. ${ }^{9}$ transgenically expressed NpHR and ChR2 H134R in zebrafish cardiomyocytes to locate the pacemaker in the developing heart, and to simulate pathological cardiac conditions like tachycardia, bradycardia, atrioventricular block, and cardiac arrest. Abilez et al. recorded photostimulation and photoinhibition of cardiomyocytes derived from human embryonic stem cells ${ }^{10}$ and human induced pluripotent stem cells ${ }^{11}$. Jia et al. ${ }^{12}$ used an all-optics system to acquire optical recordings of $\mathrm{Ca}^{2+}$ transients from tandem units of cardiomyocytes with HEK293 cells expressing ChR2. Nussinovitch et al. ${ }^{13}$ co-cultured cardiomyocytes with NIH-3T3 fibroblasts expressing ChR2, and used microelectrode arrays to monitor resynchronized electrical activity after conduction block. Kirkton et al. ${ }^{14}$ activated engineered HEK293 cells expressing ChR2 in a cardiac setting. Finally, much interest lies in the computational modeling of optogenetics control ${ }^{10,15-17}$.

In all these endeavours, an all-optics system would be ideal, combining the strength of optogenetics (precise spatiotemporal control and unipolar current injection) and optical mapping of excitability signals ${ }^{18}$ (noninvasive, high-resolution visualization of conduction patterns). Thus far, however, only $\mathrm{Ca}^{2+}$ transients have been measured in an all-optics manner ${ }^{12,19}$, whereas optical monitoring of the more fundamental voltage signals that comprise action potentials has yet to be reported for this configuration. Here, we therefore devise an apparatus that permits readouts of action-potential morphology and conduction, concurrent with orthogonal activation of optogenetic constructs expressed in NRVM monolayers. As an initial application, we explore the capability of this all-optics system to shape cardiac action-potential morphology, in particular to lengthen and shorten APDs. Such a capability might ultimately be desired to probe and manipulate pathologic conditions relating to APD abnormalities. In particular, long and short QT syndromes (LQTS or SQTS) derive respectively from unusually prolonged or abbreviated APDs, predisposing for conduction heterogeneities, ventricular arrhythmias, and sudden cardiac death ${ }^{20-22}$. The efficacy of current treatments varies considerably with underlying pathogenesis and severity of arrhythmias ${ }^{21}$, and further insight into the underlying mechanisms, spanning spatiotemporal 
scales from molecules to cell networks, would be desirable. Our alloptics system may furnish helpful tools towards this long-term goal.

\section{Results}

Biophysical properties of $\mathrm{ChR2}$ and eNpHR3.0 during actionpotential waveforms. To assess the overall feasibility of using optogenetic constructs to influence cardiac action-potential morphology, we initially assessed the magnitude of optogenetic currents using whole-cell patch-clamp of HEK293 cells stably expressing either ChR2 or eNpHR3.0. For ChR2 (Fig. 1a), we transiently activated current with pulsed blue-light illumination $(447 \mathrm{~nm})$ while voltage was held constant. In different trials, voltage was set at different levels ranging from $-80 \mathrm{mV}$ to $80 \mathrm{mV}$ in $10 \mathrm{mV}$ steps. Activation of inward current was observed between $-80 \mathrm{mV}$ to $10 \mathrm{mV}$, and current amplitude decreased as voltage became more positive. Outward current was present from $20 \mathrm{mV}$ to $80 \mathrm{mV}$, and current amplitude increased as voltage became more positive. These data are consistent with a reversal potential between $10-20 \mathrm{mV}$. In agreement with previously reported patch-clamp results ${ }^{3}$, partial inactivation of currents was clearly present. For eNpHR3.0 (Fig. 1b), currents were transiently activated by pulsed green-light illumination $(530 \mathrm{~nm})$ delivered within an analogous protocol. Light activation produced relatively uniform inward chloride currents at all voltages. More detailed inspection reveals that the chloride current increased slightly with increasing voltage, indicating that eNpHR3.0 is weakly voltage-dependent as expected from its pump-like properties. In agreement with previously reported patch-clamp results, there was no inactivation of current. Population data for ChR2 currents are shown for peak (Fig. 1c, red) and steady-state (Fig. 1c, black) current levels; these illustrate the inwardly rectifying character of this channel under physiological ionic gradients ${ }^{3,23}$. As well, population data for eNpHR3.0 currents are shown for peak (Fig. 1d, red) and steady-state (Fig. 1d, black) current levels ${ }^{2}$; these indicate the near step-like kinetics of this pump. The biophysical properties were also characterized under variable light intensities to show that net current increases with increasing light intensity for both ChR2 and eNpHR3.0 (Supplementary Fig. S2). Additionally, the inactivation kinetics of ChR2 show increasing inactivation with increasing voltage or blue-light intensity (Supplementary Fig. S3).

To further gauge the potential suitability of the optogenetic constructs for altering action-potential morphology, currents were elicited during voltage-clamp with cardiac action-potential waveforms (APW), as shown in Figures 1e and 1f. Optogenetic currents were allowed to reach steady state with respect to light exposure prior to trace onset (Figs. 1e-f, note 500-ms axis breaks in light-pulse waveform). As expected, based on the voltage step recordings, ChR2 current was greater at negative voltages (Fig. 1e) and current decreased with increasingly depolarized voltages. The overall current trace morphology was similar to the APW. Similar to the constant voltage step recordings (Fig. 1b), eNpHR3.0 current acquired during an APW (Fig. 1f) was also relatively constant, indicating little voltage-dependence of current.

Optical mapping of action potentials with orthogonal ChR2 or eNpHR3.0 excitation. A significant challenge was to record transmembrane potential via a voltage-sensitive fluorescent dye, while independently switching on/off ChR2 or eNpHR3.0. Specifically, the absorption spectrum of the more commonly used voltagesensitive dye, di-4-ANEPPS ${ }^{24}$, fully overlaps the absorption spectrum of $\mathrm{ChR}_{2}^{5}$, and partially overlaps the absorption spectrum of eNpHR3. $0^{2}$. This overlap makes it impossible to independently excite di-4-ANEPPS and the optogenetic constructs. The solution was to use the long-wavelength voltage-sensitive dye $\mathrm{PGH}^{25}$ instead, with an absorption spectrum ${ }^{26}$ separate from that of ChR2 and eNpHR3.0 (Supplementary Fig. S4).
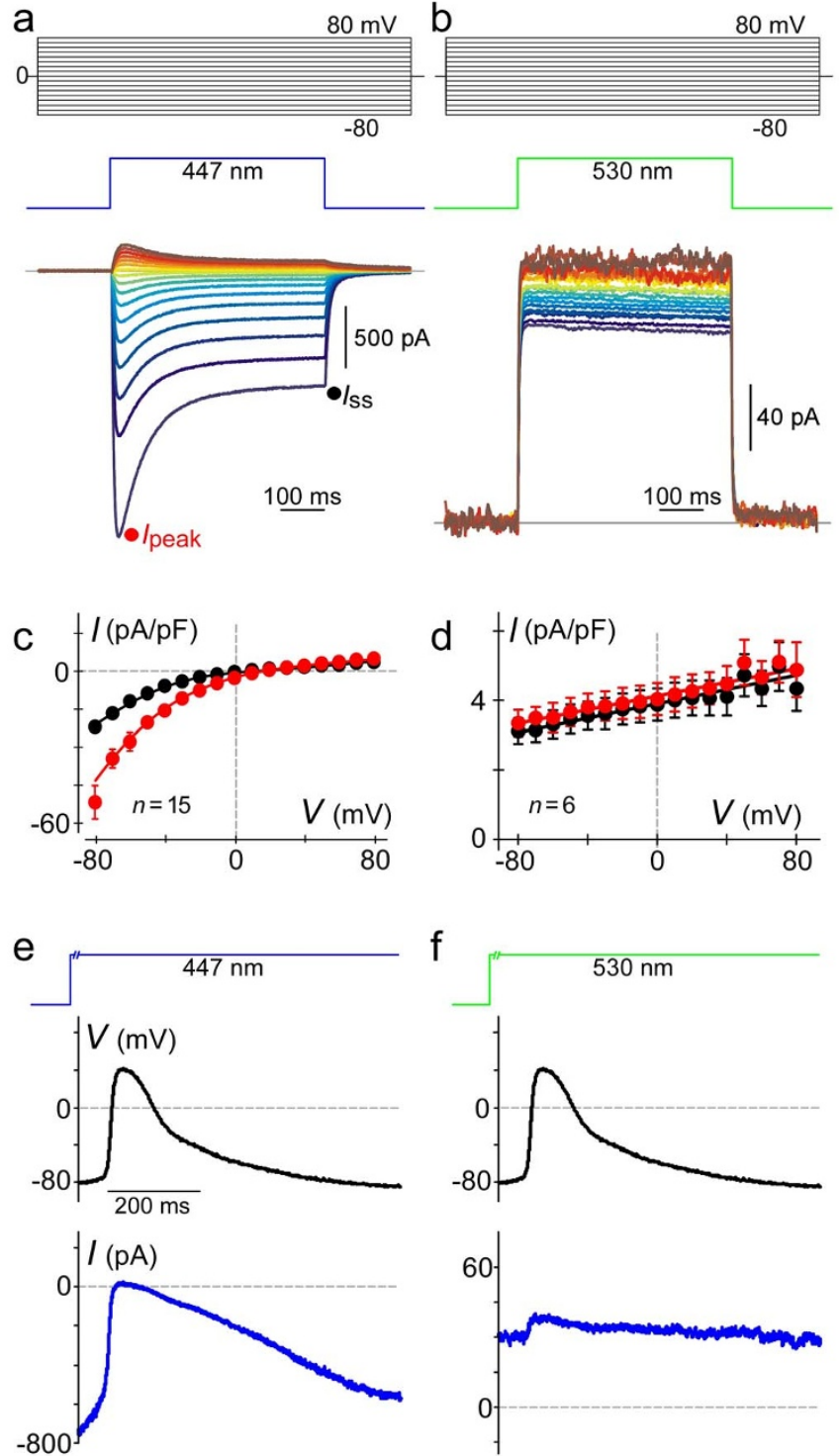

Figure $1 \mid$ Biophysical properties of optogenetic constructs and their suitability for altering excitability during action potentials. ChR2 (a, c) and eNpHR3.0 (b, d) were initially characterized at various constant levels of voltage, using whole-cell patch clamp in HEK293 cells. (a) ChR2 supports light-gated, voltage-dependent currents with a reversal potential of $\sim 17 \mathrm{mV}$ according to the curve fit of the I-V relation in (c). Partial inactivation of currents is clearly present.

(b) eNpHR3.0 is light-gated, but only weakly voltage-dependent. (c) ChR2 population data for peak (red) and steady-state (black) current levels. $n$ refers to number of cells; bars show sem. (d) eNpHR3.0 population data for peak (red) and steady-state (black) currents. $n$ refers to number of cells; bars show sem. Panels (e) and (f) examine optogenetic currents during voltage-clamp with cardiac actionpotential waveforms gauged from neonatal rat ventricular myocytes probed with PGH1 as the voltage-sensitive dye. (e) ChR2 currents during APWs are large and enhanced by repolarization. Current amplitude between the maximum during the trace onset at $-80 \mathrm{mV}$, and the minimum near the peak of the APW, was $427 \pm 159 \mathrm{pA}$ (mean \pm sem, $n=5$ cells). (f) eNpHR3.0 currents during APWs are smaller but largely independent of voltage. Current amplitude at the trace onset $32 \pm 0.4 \mathrm{pA}$ (mean \pm sem, $n=12$ cells). All blue illumination at $7.2 \mathrm{~mW} / \mathrm{mm}^{2}$. All green illumination at $1.4 \mathrm{~mW} / \mathrm{mm}^{2}$. Axis breaks $(500 \mathrm{~ms})$ in light-power schematics atop (e) and (f), ensure that channels had reached near steady-state (in)activation with respect to light, prior to trace onset. 


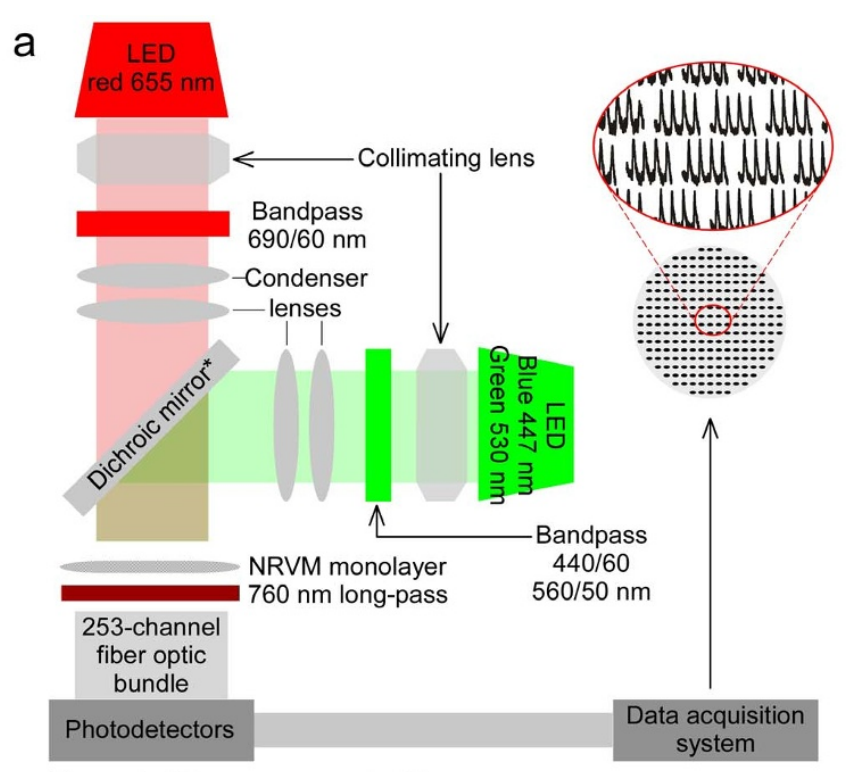

*blue mode $475 \mathrm{~nm}$ or green mode $618 \mathrm{~nm}$
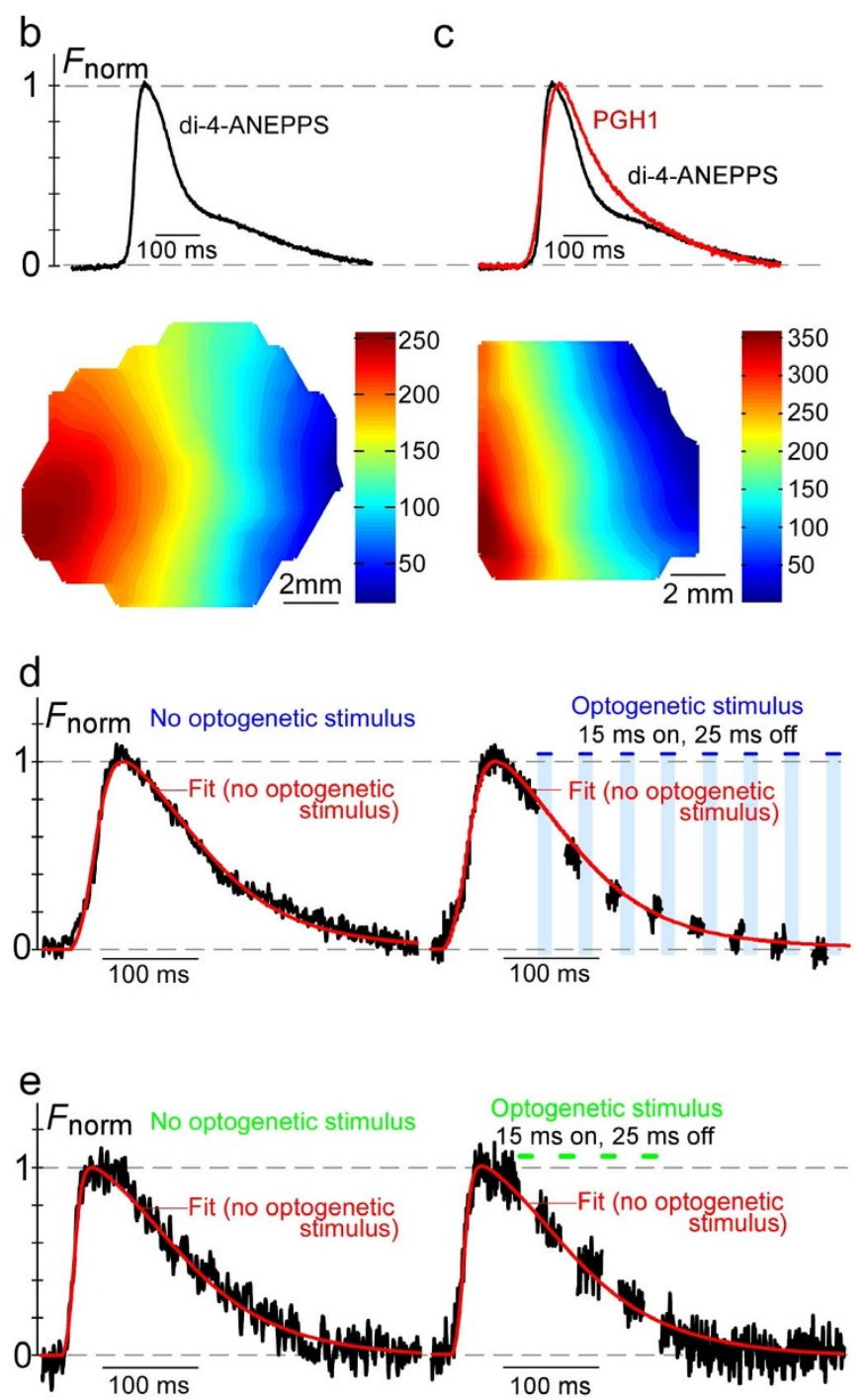

Figure $2 \mid$ Optical mapping system featuring detection of red-shifted PGH1 voltage-sensitive dye signals, concurrent with orthogonal excitation of either ChR2 or eNpHR3.0 optogenetic constructs. (a) Optical layout with dual-pathway excitation modules placed above NRVM monolayer. Fluorescence emission is captured from below, through a 253-channel fiber optic bundle. (b) Top, Exemplar NRVM (without optogenetic expression) action potential averaged from multiple channels, using customary di-4-ANEPPS voltage-sensitive dye. Bottom, corresponding activation map of uniform propagation. The color bar represents activation time in ms defined as the time of the stimulus to $50 \%$ of peak. (c) Top, Action-potential morphology was largely maintained with use of the red-shifted PGH1 dye. APDs were modestly prolonged compared to di-4-ANEPPS. Bottom, Propagation with PGH1 was also uniform throughout the monolayer, though PGH1-stained monolayers exhibited somewhat slower conduction. (d, e) Blue- or green-light pulses had no collateral effects on NRVMs lacking optogenetic expression, as demonstrated by exemplar PGH1 traces from a single recording channel. Blue or green illumination caused baseline shifts of the fluorescence signal, requiring crosstalk compensation, which is described in more detail in Method "Gap compensation for blue- and green-light bleed-through." All blue illumination at $7.2 \mathrm{~mW} / \mathrm{mm}^{2}$. All green illumination at $1.4 \mathrm{~mW} / \mathrm{mm}^{2}$.

An optical mapping system ${ }^{18}$ was engineered (Fig. 2a) to enable detection of red-shifted PGH1 dye signals ${ }^{25}$ concurrent with orthogonal excitation of either ChR2 or eNpHR3.0. NRVM monolayers stained with PGH1 were directly illuminated using a red $(655 \mathrm{~nm})$ LED array passed through an interference filter $(690 / 60 \mathrm{~nm})$. Action potentials, in the form of fluorescence emission, were acquired at wavelengths above $760 \mathrm{~nm}$ using a long-pass filter and a 253-channel fiber-optic bundle. To excite ChR2, light from a perpendicularly aligned blue (447 $\mathrm{nm}$ ) LED array was passed through an interference filter $(440 / 60 \mathrm{~nm})$, and directed onto the monolayer using a dichroic mirror splitting at $475 \mathrm{~nm}$. To excite eNpHR3.0, light from a perpendicularly aligned green $(530 \mathrm{~nm})$ LED array was passed through an interference filter $(560 / 50 \mathrm{~nm})$, and directed onto the monolayer using a dichroic mirror splitting at $618 \mathrm{~nm}$. The resulting data features multiple channels of action-potential data. Each channel reports the signal of a single fiber-optic, yielding a composite action potential from many syncytial myocytes in a 1-mm diameter spot of the monolayer.

To verify that PGH1 does not substantially affect action-potential morphology, spatially averaged action potentials acquired with the traditionally used di-4-ANEPPS (Figs. 2b top and 2c top, black) were compared to those obtained with PGH1 (Fig. 2c top, red). Overall, action potentials measured with PGH1 were similar to those observed with di-4-ANEPPS, with PGH1 responses exhibiting slightly longer APD and modestly slower depolarization/repolarization rates. Activation isochrone maps with di-4-ANEPPS (Fig. 2b bottom) and PGH1 (Fig. 2c bottom) both indicated uniform propagation, with possibly slower conduction velocity measured using PGH1. This slight slowing of action-potential upstroke and downstroke observed with PGH1, along with a somewhat diminished conduction velocity, may relate to the higher levels of PGH1 staining required to achieve an adequate signal-to-noise ratio. Nonetheless, these effects were small enough to proceed with the use of PGH1 in the experiments that follow.

To confirm that blue or green illumination for optogenetic stimulation would not in itself interfere with action-potential responses recorded by PGH1, NRVM monolayers lacking optogenetic constructs were stained with PGH1 and electrically paced. Action potentials were then recorded without (Fig. 2d left) and with (right) delivery of multiple blue- or green-light pulses. As baseline, the control response on the left (black trace) was fitted with the smooth red function for convenient comparison. Multiple high-intensity bluelight pulses introduced bleed-through artifacts manifest as gaps in the black trace on the right (see Methods "Gap compensation ..."; and Supplementary Fig. S1). Nonetheless, superposition of the smooth red curve fit from control indicates that the blue pulses did 

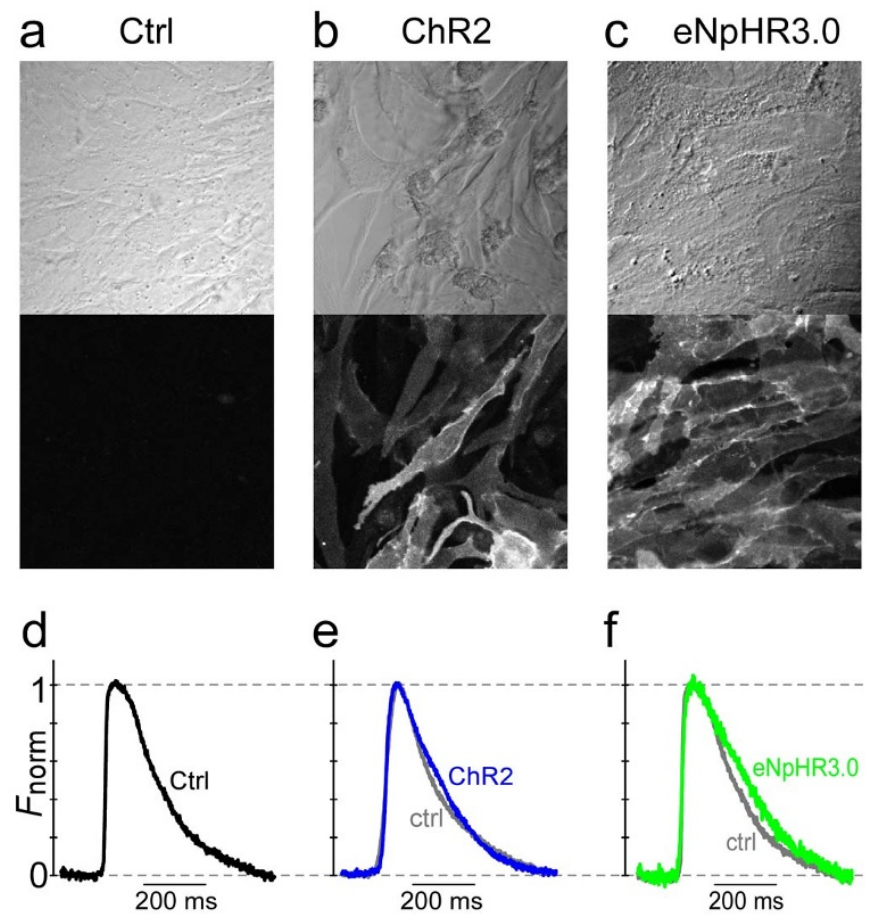

Figure $3 \mid$ Transduction of NRVM monolayers with lentiviruses bearing ChR2 or eNpHR3.0 did not appreciably perturb action-potential morphology from control (absent optogenetic stimulation with blue or green light). (a, d) Control NRVMs without viral transduction.

(a) Transmission (top) and fluorescence (bottom) confocal images.

(d) Action potentials were averaged from multiple channels of optical mapping data, with PGH1 as dye. Parallel culture to those transduced with lentiviruses in later panels. (b, e) Results for NRVM transduced with lentivirus bearing ChR2; format as in (a). (b) Fluorescence is from YFP fused to ChR2, and reflects expected membrane targeting of ChR2.

(e) Action potential (blue) essentially unchanged from control (gray). (c, f) Results for NRVM transduced with lentivirus bearing eNpHR3.0; format as in (a). (c) Fluorescence is from YFP fused to eNpHR3.0, and reflects membrane targeting of optogenetic construct. (f) Action potential (green) essentially unchanged from control (gray), as copied from panel (a).

not by themselves affect action-potential morphology. Likewise, multiple high-intensity green-light pulses did not by themselves affect action-potential morphology (Fig. 2e).

Un-stimulated ChR2 or eNpHR3.0 minimally alters cardiac action potentials. To exclude artifactual changes in action-potential morphology by expression of optogenetic constructs in the absence of their activation, we compared NRVM monolayers in the absence of lentiviral transduction (Fig. 3a) with those transduced with lentivirus bearing ChR2 (Fig. 3b) or eNpHR3.0 (Fig. 3c). Under optical mapping using $\mathrm{PGH} 1$, averaged action potentials for the un-transduced monolayers displayed their usual morphology as shown in Figure 3d. Throughout, no blue or green illumination was applied to exclude activation of optogenetic constructs. Infection of NRVM monolayers with lentivirus bearing ChR2 resulted in widespread expression across cells, each showing the expected membrane targeting of YFPtagged channels (Fig. 3b, bottom panel). The spatially averaged action potential (Fig. 3e, blue) from the same culture and infection indicates no appreciable perturbation of action-potential morphology from control (Fig. 3e, gray trace). Similarly, infection of NRVM monolayers with lentivirus bearing eNpHR3.0 was efficient and showed membrane targeting of the YFP-tagged pumps (Fig. 3c, bottom panel). The spatially averaged action potential (Fig. 3f, green) also suggests minimal perturbation of action-potential morphology from control (Fig. 3f, gray trace, reproduced from Fig. 3d). Infection with empty virus (only GFP) also did not affect actionpotential morphology (Supplementary Fig. S5). Thus, expression of optogenetic constructs did not in themselves alter action-potential behavior.

Functional excitation or silencing of NRVM monolayers with ChR2 or eNpHR3.0. We initially tested for simple stimulation and silencing of action potentials by brief activation of the optogenetic constructs. Voltage was monitored with optical mapping utilizing PGH1. In monolayers transduced with ChR2, action potentials were elicited by a train of blue-light pulses (51 ms duration) at maximal power $\left(7.2 \mathrm{~mW} / \mathrm{mm}^{2}\right)$ with no electrical stimulation (Fig. 4a, left). The intensity and duration of the blue-light pulses were long enough to simultaneously excite the entire monolayer, preventing propagation (Fig. 4a, right). Accompanying manipulation of monolayer contraction was also observed (Supplementary Movie 1 shows that a monolayer bearing ChR2 contracts after short-duration, high-intensity blue-light pulses). When the bluelight pulse intensity was lowered or the duration was shortened, propagation was evident across the monolayer (data not shown). In eNpHR3.0-expressing NRVMs, electrically paced action potentials were silenced with variable intensity green-light pulses (Fig. 4b, top). The green-light intensities (Fig. 4b, green trace, in $\mathrm{mW} / \mathrm{mm}^{2}$ ) were $1.4,1.4,1.24,0.67,0.14,1.4$, and 1.4. Electrical stimulation nicely induced a propagating depolarizing wave from left to right (Fig. 4b, bottom, leftmost and rightmost optical maps). The first four green-light pulses successfully abolished the electrically paced action potentials, also evident across space by the lack of activation in the isochrone map (Fig. 4b, bottom, optical map second from left). However, with each of these four successful silencing events, an action potential was elicited shortly after cessation of the green-light pulse, much like an anode-break-like response. As might be expected, this break-like response produced nearly uniform excitation across the preparation (Fig. 4b, bottom, map third from the left). Analogous manipulation of monolayer contraction was also observed (Supplementary Movie 2). The fifth, low intensity green-light pulse failed to silence the electrically paced action potential, hinting at the possibility of tuning green-light intensity to do more than simply switch off electrically stimulated responses, an issue that will be explored two sections below.

APD elongation using multiple blue-light pulses in ChR2-expressing monolayers. Up to this point, we have demonstrated the ability to record action potentials under an optical mapping configuration permitting orthogonal excitation of optogenetic constructs, reproducing previously reported optogenetic phenomena in cardiac preparations. Here, we directly test whether ChR2 may be used to sculpt action-potential morphology itself. We sought to increase APD using blue-light excitation in ChR2-expressing monolayers. First, to verify that APDs can be increased appreciably in NRVMs, and to set a heuristic target for optogenetic APD elongation, we performed optical mapping with PGH1 on monolayers incubated in $1 \mu \mathrm{M}$ Bay K 8644, an L-type calcium channel agonist ${ }^{27}$. Figure $5 \mathrm{a}$ indicates substantial augmentation of APD, as illustrated by comparison of the spatially averaged action potential obtained under pharmacological enhancement of L-type current (Fig. 5a, right, black trace) to that of control action potentials (Fig. 5a left, and right gray trace). Conversely, a calcium channel antagonist shortened APD (Supplementary Fig. S6). To test for elongation of APD by ChR2, increasing numbers of punctuated blue-light pulses $\left(7.2 \mathrm{~mW} / \mathrm{mm}^{2}\right)$ were administered during the repolarization phase of electrically paced action potentials in a monolayer expressing ChR2 (Fig. 5b, i-iv). Responses without blue-light stimulation (o) were interspersed to confirm the return to a stable baseline between presumed perturbations. Gaps indicate periods of blue-light bleed- 

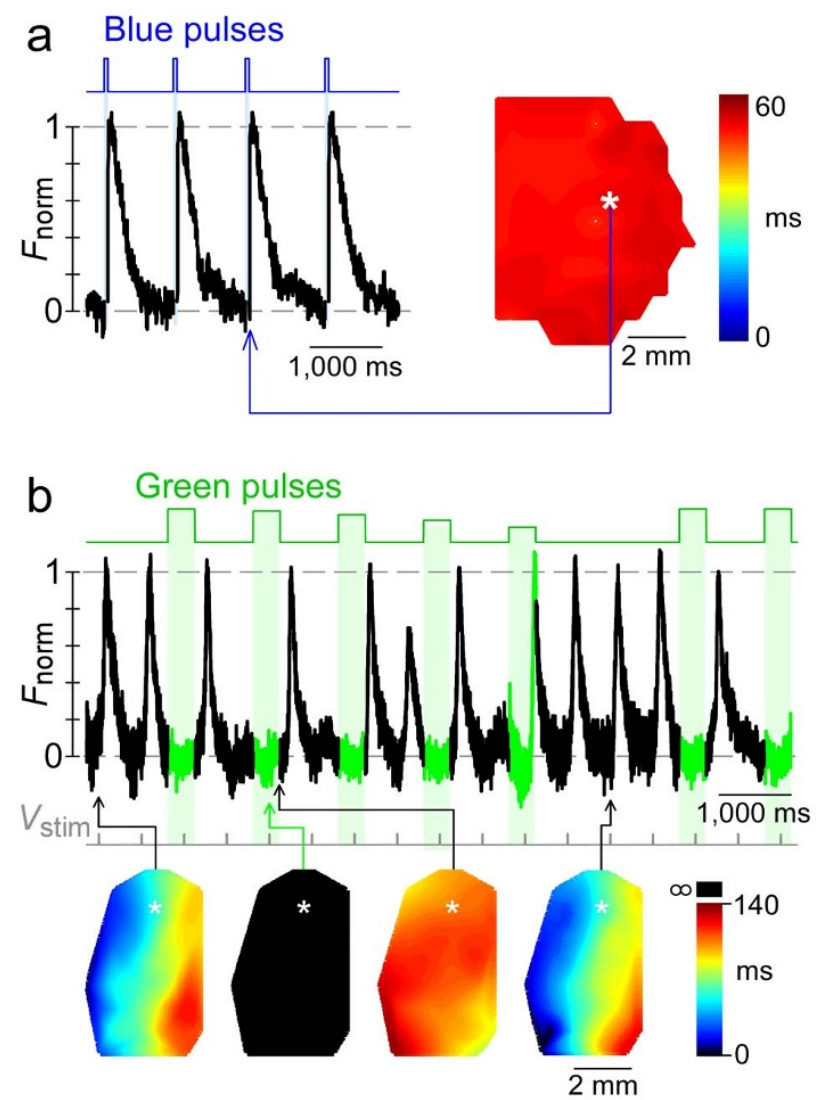

Figure $4 \mid \mathrm{ChR} 2$ and eNpHR3.0 can respectively trigger and silence action potentials, as seen from optical mapping data obtained with PGH1. (a) Exemplar result of NRVM monolayer expressing ChR2 exposed to high-intensity blue pulses (51 ms, top, blue trace), without electrical stimulation. Hardly noticeable gaps in records reflect periods of blue-light bleed-through. Excitation of the monolayer occurs simultaneously with no propagation. Time zero in the activation isochrone map corresponds to the start of the blue pulse. Position of the exemplar trace is marked with a white asterisk on the isochrone map. (b) In eNpHR3.0-expressing NRVMs, electrically paced action potentials can be silenced with variable intensity green pulses (top, green trace). The first four green high-intensity pulses silence action potentials but evoke anode-break-like action potentials upon cessation of green pulses. Electrical pacing in gray (just below fluorescence trace). Bottom, isochrone maps corresponding to various phases of trace as shown. Propagated depolarizing wave for electrically stimulated responses (leftmost and rightmost maps). No excitation across monolayer during the second silencing green pulse (second from left map). Non-propagating uniform excitation in break-like response (third from left map). Zero time of maps is instant of electrical stimulus, except for break-like response where cessation of green-light pulse is taken as time zero. Position of recording site for exemplar trace is marked with a white asterisk on the isochrone maps.

through (see Methods "Gap compensation ..."). Even on the comparatively compressed timescale of Fig. 5b, APD prolongation during blue-light stimulation is evident. To confirm this impression, Fig. $5 \mathrm{c}$ aligns the various APWs on an expanded time-base, and fits to these waveforms (smooth red curves) are overlaid to improve visual comparison. To improve precision, fits were performed sequentially on trials with increasing number of blue pulses (see Methods "Gap compensation ..."). Seen in this manner, each additional blue-light pulse induced further augmentation of APD, as shown by the shaded red region denoting the difference between blue-light-stimulated waveforms and control. Alignment of smooth fits to the various responses, shown at the bottom right, underscores the graded manner in which high-intensity blue-light pulses can elongate APD in a graded fashion. Indeed, the waveform observed with four blue-light pulses (Fig. 5d, red trace) approximates the elongation produced by our heuristic maneuver with $1 \mu \mathrm{M}$ Bay $\mathrm{K}$ 8644 (black trace). These exemplar trends were fully corroborated by $\mathrm{APD}_{80}$ population data from multiple optical channels on a single coverslip (Fig. 5e), and by $\mathrm{APD}_{80}$ data averaged over multiple coverslips (Fig. 5f). Notably, the $\mathrm{APD}_{80}$ measurements were made on directly present portions of the data that did not require smoothfunction fits. Thus, these latter analyses (Figs. 5e-f) thereby confirm the ability to optogenetically sculpt APD elongation by delivery of blue pulses.

APD shortening using green-light pulses in eNpHR3.0-expressing monolayers. To explore the counterpoint of the previous experiments, this section tests for the possible shortening of action potentials by activation of eNpHR3.0 in NRVM monolayers. In this regard, while high-intensity green light could activate eNpHR3.0 to such an extent that action potentials were altogether silenced (Fig. 4b), we wondered whether lower-intensity green-light activation might allow for responses with attenuated duration. Accordingly, we scrutinized electrically stimulated responses of NRVMs, particularly during modest activation of eNpHR3.0 (Fig. 6a). Green-light intensities in this panel were 1.4, 1.4, 1.24, $0.67,0.14$, and $1.4\left(\mathrm{~mW} / \mathrm{mm}^{2}\right)$. Similar to Fig. $4 \mathrm{~b}$, higher intensity green-light pulses altogether abolished action potentials. However, during the weaker green-light pulses $\left(0.67\right.$ and $\left.0.14 \mathrm{~mW} / \mathrm{mm}^{2}\right)$, miniature action potentials with blunted amplitude and shortened durations were observed (Fig. 6a, green asterisk). Figure $6 \mathrm{~b}$ displays a close-up comparison of a control action potential (Fig. 6a, black asterisk) and a miniature action potential (Fig. 6a, green asterisk). Clearly evident are the action potential morphology changes wrought by activating eNpHR3.0 with green light: a diminished amplitude and shortened duration at the $80 \%$ level (red dashed line). Population data for APDs averaged across multiple channels on a single coverslip (Fig. 6c) verified that the shortened and blunted action-potential morphology, as induced by modest green light, was present across the monolayer. Curiously, if green pulses were selectively delivered during the repolarizing phase of action potentials, as was done with ChR2 in Figure $5 \mathrm{~b}$, action potentials could not be shortened. Presumably, the expression of eNpHR3.0 current was insufficient to perturb action-potential trajectories once native voltage-activated channels become fully activated by larger depolarizations. Nonetheless, these results indicate the potential for optogenetic hyperpolarizing currents to induce shorter action potentials, particularly if larger eNpHR3.0 currents could be produced.

\section{Discussion}

Our work achieves a significant technical advance in cardiac optogenetics-the ability to optically map voltage signals underlying action-potential and conduction profiles in cardiac monolayers, while simultaneously modulating the observed action potentials via optogenetics. In particular, using an all-optics setup, we show that cardiac APD can be increased in a graded manner by activating ChR2 currents, and action-potential amplitude and duration can be downwardly modulated by activating eNpHR3.0 currents. By contrast, the bulk of prior optogenetic effort in the heart has focused on outright excitation or silencing of activity ${ }^{7-10,12}$. As well, when measured, conduction profiles were obtained with a micro-electrode array $^{7}$ or by optical mapping of calcium transients ${ }^{12}$. Instead, our work here demonstrates that it is possible to acquire transmembrane potentials in this context, promising future opportunities to combine simultaneous recordings of voltage and $\mathrm{Ca}^{2+}$ with orthogonal excitation of ChR2 and eNpHR3.0.

One potential limitation might concern the use of ChR2 to prolong APDs in preparations where the plateau phase (phase 2) is more 

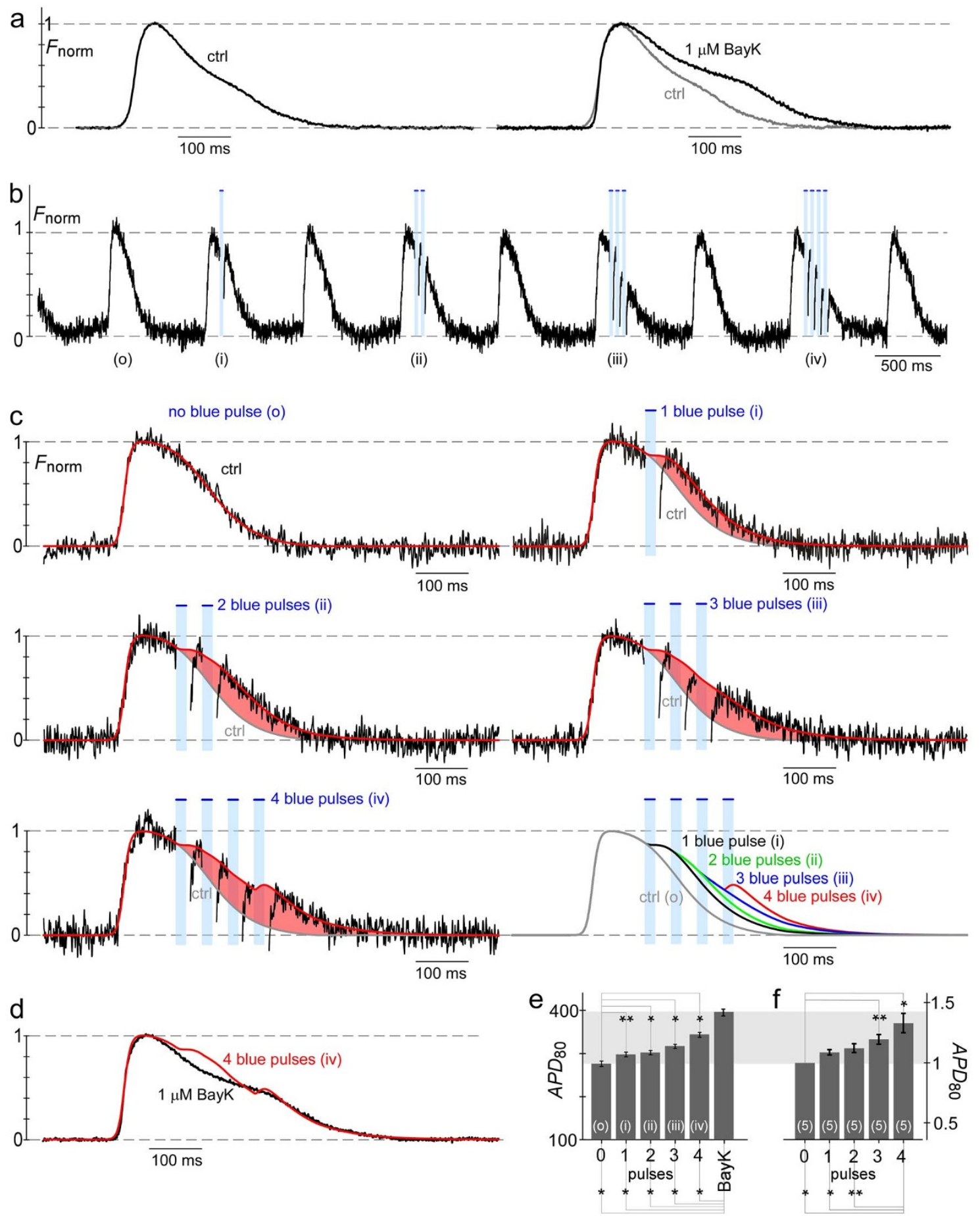

Figure $5 \mid$ APD can be increased in ChR2-transduced NRVMs, using multiple blue pulses (20 ms on, $30 \mathrm{~ms}$ off) delivered during the repolarization phase. (a) Action potential of control NRVMs (left black trace, without ChR2) can be elongated by $1 \mu$ M Bay K 8644 (right black trace; control reproduced as gray trace), demonstrating pharmacological APD prolongation. (b) Exemplar trace demonstrating APD lengthening by transient ChR2 activation in NRVMs. Action potentials with increasing numbers of blue pulses (i-iv, blue bars) interspersed with control APs (o). Trace shows gaps due to crosstalk compensation of the baseline shifts during blue illumination. (c) Expanded time-base display of records in (b). APD increases in a graded manner with number of blue pulses. All action potentials (control (o) and blue pulse action potentials (i-iv)) were fitted (red) to facilitate visualization after crosstalk compensation of blue-light pulses. Right bottom, comparison of fits. (d) APD with four blue pulses (iv) is significantly increased to resemble $1 \mu \mathrm{M}$ Bay K 8644 action potential. (e) Bar graphs of average $\mathrm{APD}_{80}$ for various types of action potentials, confirming a graded increase in $\mathrm{APD}$ with increasing blue-pulse number (mean \pm sem, $n=16$ channels from single coverslip). The four-blue-pulse APD ${ }_{80}$ approximates pharmacologically lengthened $\mathrm{APD}_{80}$ observed with $1 \mu \mathrm{M}$ Bay K 8644 (mean \pm sem, $n=82$ channels). Analogous results for Bay K 8644 were obtained in a total of $n=9$ coverslips. Statistical significance computed via 1-way ANOVA with Tukey-Kramer multicomparison criterion, with $* *$ signifying $p<0.05$ and $*$ signifying $p<0.01$. (f) Similar APD trends were confimed for population analysis over $n=5$ coverslips (parentheses), where APD 80 values were normalized to values obtained from responses without a blue-light pulse. Statistical significance computed via 1-way ANOVA with Tukey-Kramer multicomparison criterion, with ${ }^{* *}$ signifying $p<0.05$ and $*$ signifying $p<0.01$. 

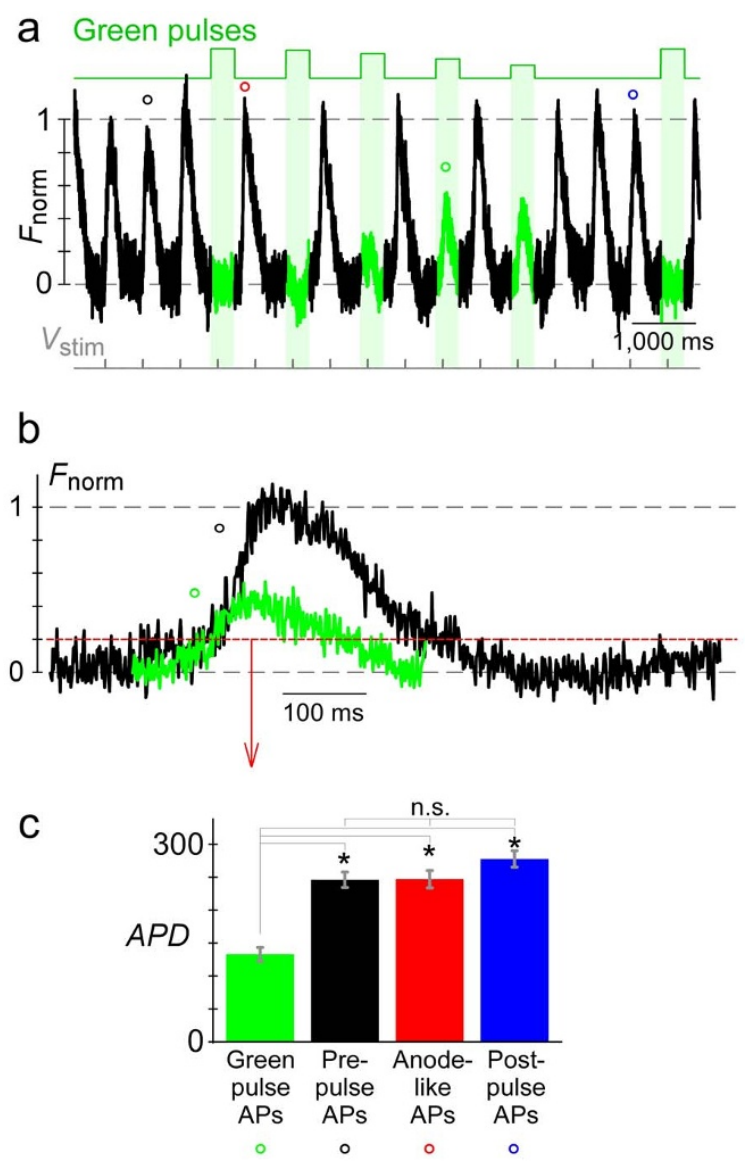

Figure 6 | Electrically stimulated action potentials become shorter and smaller in amplitude during low-intensity green pulses delivered to NRVMs expressing eNpHR3.0. (a) Effects of variable intensity green pulses (top). Electrical stimuli (bottom). Reference 'pre-pulse' response (black asterisk) is electrically paced. Higher intensity green pulses (first two) silence action potentials. Lower intensity pulse (green asterisk) yields a short duration and smaller amplitude action potential. Anode-break-like response (red asterisk). Post-green pulse (blue asterisk) shows return to control. (b) Closer view of control (black asterisk) compared to low intensity green-pulse action potential (green asterisk). (c) APD metrics (mean \pm sem, $n=12$ channels from single coverslip), confirming APD shortening by low intensity green-light pulses (green asterisk). Green pulse APD (green asterisk) calculated at $\mathrm{APD}_{80}$ of control ((b), red dashed line) without normalization. Statistical significance computed via 1-way ANOVA with Tukey-Kramer multicomparison criterion, with * signifying $p<0.01$. n.s. signifies $p>0.25$.

pronounced (e.g., humans, rabbits, and guinea pigs) than in the rat NRVMs used here (similar to mouse). Because ChR2 current reverses at about 10-20 mV (Figure 1C), one might wonder whether the resulting small magnitude of ChR2 currents during a human-like plateau phase (near $10-20 \mathrm{mV}$ ) would preclude appreciable APD prolongation by $\mathrm{ChR} 2$ in this setting. However, it is worth considering how $I_{\mathrm{K} 1}$, which manifests diminutive currents near its reversal potential at $E_{\mathrm{K}}$, nonetheless anchors the cellular resting membrane potential near its reversal potential. Moreover, the magnitude of native currents and conducatance during the plateau phase of human-like action potentials would be relatively small ${ }^{28}$, so ChR2 need overcome only moderately sized native currents to prolong the plateau phase of these action potentials. Favoring this outcome on empirical grounds is the ability of the 'first blue light pulse' in Figure 5C to prolong the NRVM action potential. In particular, the absolute potential at this point of the action potential would be greater than $0 \mathrm{mV}^{29,30}$. Thus, we would anticipate that ChR2 could still prove effective at manipulating action potentials in preparations manifesting a more pronounced plateau phase.

A more stringent limitation concerns practical limits on the levels of eNpHR3.0 current that could be expressed in NRVMs. In particular, action potential duration and amplitude could be attenuated only by commencing modest activation of eNpHR3.0 prior to an electrical stimulus pulse, and continuing this activation throughout a subsequent action potential. Notably, we were unsuccessful in altering action potential shape by introducing green-light pulses only during the plateau or repolarizing phases of action potential, as was accomplished with ChR2. This curious dichotomy likely arises because the magnitude of hyperpolarizing photocurrent required to blunt actionpotential waveforms is less if action potentials are attenuated prior to their full evolution. Delivery of modest hyperpolarizing photocurrent prior to an electrical stimulus may allow the voltage threshold for eliciting an action potential to be reached, but this delivery may still limit the ensuing extent of voltage activation of $I_{\mathrm{Na}}$ and $I_{\mathrm{Ca}, \mathrm{L}}$ currents, which in turn influences action-potential morphology. By contrast, once a 'normal' action potential reaches peak amplitude, comparatively larger $I_{\mathrm{Na}}$ and $I_{\mathrm{Ca}, \mathrm{L}}$ currents must be dealt with, and somewhat larger repolarizing $I_{\mathrm{Ks}}$ and $I_{\mathrm{Kr}}$ currents may dwarf the relative contribution of repolarizing photocurrents achievable by eNpHR3.0 (Appendix B). Unfortunately, we could not produce larger optogenetic hyperpolarizing currents, because eNpHR3.0 currents were smaller to start (cf., Fig. If in HEK293 cells), and eNpHR3.0 also seemed more toxic to myocytes than was ChR2. An alternate hyperpolarizing photocurrent, Archaerhodopsin ${ }^{31}$, was tested but currents were yet smaller in NRVMs.

Another current challenge regards the finite spectral range within which the dyes and optogenetic constructs must function. For the near future, the issues of spectral crosstalk will be resolved by the development of better performing voltage-sensitive dyes with absorption spectra at wavelengths outside the blue and green range $^{26,32}$. Improved genetically-encoded voltage indicators are also now becoming available ${ }^{33-35}$. Furthermore, the toolbox of optogenetic constructs continues to grow, diversifying the types of lightactivated control available for basic science research ${ }^{36}$. As for the limitations of eNpHR3.0 current amplitudes, one simple improvement would be to deliver greater green-light activation; the maximum green-light power we were able to achieve via commercial LED components was $\sim 1.4 \mathrm{~mW} / \mathrm{mm}^{2}$, and our characterization of eNpHR3.0 indicates that further eNpHR3.0 current could be recruited with greater green-light excitation (Supplementary Fig. S2). Another potential improvement would be to use transgenic mice or rats bearing the optogenetic constructs, thus offering potentially uniform and highly expressing optogenetic constructs across myocytes.

More broadly, multiple exciting applications loom on the horizon. Firstly, with regard to arrhythmia research, a next-generation version of our apparatus using digital micromirror devices ${ }^{9}$ could create and study customized time-varying spatial patterns to investigate how local geometry interacts with cellular excitability to promote or inhibit reentrant waves. The flexibility and power of such a strategy are enormous. Secondly, another relevant dimension of arrhythmogenesis concerns how many cells must manifest ectopy or altered QT properties, and in what distribution, to induce appreciable arrhythmogenic behavior at the whole-heart and cell-monolayer level. This question has received much attention from the computational biology community ${ }^{37-39}$, but means of experimental verification are at present limited ${ }^{37,38}$. Optogenetic approaches to trigger ectopy or altered action-potential morphology in variable numbers of cells, with differing proximity and distribution, are likely to weigh importantly, much as optogenetics has commented on the minimal number of neurons required to form memories ${ }^{40}$. Thirdly, ChR2 promises precision prolongation at certain points of the action potential waveform, a feature difficult to achieve by injection of external currents, or 
by manipulating the complement or behavior of ion channels. This capability is particularly well suited to testing for the existence of critical windows for inducing proarrhythmic behavior, such as early afterdepolarizations in a monolayer settings ${ }^{41}$. Fourthly, optogenetic approaches offer new means to explore activity-dependent signaling in heart. Rapid electrical pacing can induce pathological changes leading to heart failure ${ }^{42}$. Moreover, electrical resynchronization therapy can do much to revert myocytes from failing hearts towards a normalized profile characteristic of healthy hearts, according to a 'reverse remodeling' process ${ }^{43}$. As well, activity-dependent signaling is believed to bias stem cells towards distinct cardiac lineages ${ }^{44}$, and to coordinate development of embryonic and neonatal heart ${ }^{45}$. The complex interaction of activity with multiple signaling pathways and genetic changes likely underlies these alterations in cellular phenotype ${ }^{46-50}$. Optogenetic control of myocytes offers new flexibility in specifying the activity profile of various preparations. Not only can excitable myocytes be altogether triggered or silenced, pre-excitable cells can also be depolarized in controllable patterns, and the shape of action potentials in excitable cells adjusted. One intriguing signaling hypothesis is that changes in voltage itself (rather than ensuing alterations in calcium signaling) may trigger key pathways; optogenetic control during calcium channel blockade may prove revealing in this regard. Finally, optocurrents might ultimately be exploited as a next generation implantable antiarrhythmic device ${ }^{16,51}$, if means could be achieved to safely express optogenetic constructs in the heart. In this regard, it would be intriguing to drive expression of optogenetic constructs with promoters that are preferentially activated in regions of pathology. Alternatively, injection of cardiac stem cells bearing optogenetic constructs could preferentially target regions of myocardial damage ${ }^{52,53}$. In all, the opportunities are vast and exciting, and should continue to draw creative minds to this field.

\section{Methods}

NRVM isolation. NRVMs were isolated as reported ${ }^{18}$. Briefly, ventricles of P2 Sprague-Dawley rats (Harlan) were excised and incubated $\left(\sim 16 \mathrm{hr}, 4^{\circ} \mathrm{C}\right.$ shaking water bath) in $50 \mathrm{~mL} \mathrm{HBSS}$ (Gibco) containing $10 \mathrm{mg}$ trypsin ( 597,000 USP units/ $\mathrm{g}$, Amersham Life Sciences). The next day, single ventricular myocytes were isolated by multiple rounds of mechanical shaking in $50 \mathrm{~mL}$ HBSS containing $50 \mathrm{mg}$ collagenase Type 2 (215 units/mg, Worthington). Cells were filtered through a $70 \mu \mathrm{m}$ strainer (Falcon), and individual cells were re-suspended in M-199 medium (Gibco) containing 10\% FBS (Gibco), 2 mM L-glutamine (Gibco), $19.4 \mathrm{mM}$ glucose (Sigma), $100 \mu \mathrm{M}$ MEM non-essential amino acids (Gibco), $10 \mathrm{mM}$ HEPES (Invitrogen), $1.967 \mu \mathrm{M}$ Vitamin B12 (Sigma), and $12.84 \mu \mathrm{M}$ penicillin-G (1555 unit/mg, Sigma). The cells were successively plated in T75 and T175 flasks and incubated for $1 \mathrm{~h}$ each. Myocyte monolayers were cultured on plastic coverslips (McMaster, 85585k103) precoated with $2.5 \mu \mathrm{g} / \mu \mathrm{L}$ fibronectin (Invitrogen), $2.5 \times 10^{5}$ cells per $4.8-\mathrm{mm}$ diameter or $5 \times 10^{5}$ cells per 10 -mm diameter coverslip.

Molecular biology. For cardiac expression, eNpHR3.0 from pLenti-hSyneNpHR3.0-eYFP (from K. Deisseroth, Stanford University) was cloned into pPTCMV-eGFP (from G. Tomaselli, Johns Hopkins University) and designated pPTCMV-eNpHR3.0-eYFP.

Lentivirus production and transduction. Lentiviruses were produced according to modified, published protocols ${ }^{54}$ using the calcium-phosphate method. For ChR2 lentivirus production, HEK293T cells were transfected with $20 \mu \mathrm{g}$ pLenti-EF1alphaChR2-YFP-WPRE (pLECYT), $10 \mu \mathrm{g}$ deltaR8.74, and $10 \mu \mathrm{g}$ VSVG (all from K. Deisseroth, Stanford University) per $15-\mathrm{cm}$ plate. For eNpHR3.0 lentivirus production, $20 \mu \mathrm{g}$ pPT-CMV-eNpHR3.0-eYFP, $7 \mu \mathrm{g}$ pMDLg-pRRE, $10 \mu \mathrm{g}$ pMD2.G, and $5 \mu \mathrm{g}$ of pRSV-Rev (all from G. Tomaselli, Johns Hopkins University) were transfected per $15-\mathrm{cm}$ plate. Virus was concentrated from supernatant using the ultracentrifuge method and stored in $15 \mathrm{uL}$ aliquots at $-80^{\circ} \mathrm{C}$. Titre was determined by transducing HEK293 cells in 10-fold serial dilutions and checking for fluorescence $72 \mathrm{~h}$ later. For optical mapping, NRVM monolayers were transduced with either ChR2 $\left(\sim 4 \mu \mathrm{L}, 1 \times 10^{7} \mathrm{pfu} / \mathrm{mL}\right)$ or eNpHR3.0 lentivirus $\left(\sim 5 \mu \mathrm{L}, 1 \times 10^{10} \mathrm{pfu} / \mathrm{mL}\right)$. We did not find obvious signs that expression of either ChR2 or eNpHR3.0 affected resting potentials. In particular, action potential morphologies were largely unchanged (Figure 3D-F), arguing against appreciable depolarization resulting in decreased $\mathrm{Na}$ channel availability. Moreover, threshold voltages required for capturing preparations were unchanged with opsin expression (no opsin, $11.88 \pm$ $2.37 \mathrm{~V}(n=30$ coverslips); halorhodopsin, $11.74 \pm 2.74 \mathrm{~V}(n=35$ coverslips $)$; all n.s.), again suggesting maintained $\mathrm{Na}$ channel availability.
Optical mapping. Optical mapping studies were performed on NRVM monolayers 8-10 days post-plating, using di-4-ANEPPS ${ }^{18}$ (Fig. 2b) or PGH1 (all other mapping data). Coverslips of monolayers were placed in a custom-designed chamber and continuously superfused with warm $\left(36 \pm 0.5^{\circ} \mathrm{C}\right)$ Tyrode's solution (in $\left.\mathrm{mM}\right): 135$ $\mathrm{NaCl}, 5.4 \mathrm{KCl}, 1.8 \mathrm{CaCl}_{2}, 1 \mathrm{MgCl}_{2} \cdot \mathrm{H}_{2} \mathrm{O}, 0.33 \mathrm{NaH}_{2} \mathrm{PO}_{4}, 5$ HEPES, 5 glucose, $\mathrm{pH}=$ 7.4, $300 \mathrm{mOsm}$. Cells were stained with voltage-sensitive dye, and a Plexiglas cover placed over the chamber to minimize motion artifact from solution flow. Field stimulation was delivered by 2 parallel platinum line electrodes $2.7 \mathrm{~cm}$ apart. Transmembrane potentials visualized as optical fluorescent signals were collected directly below the chamber with an array of 253 optic fibers ( $1 \mathrm{~mm}$ diameter each) arranged in a $17-\mathrm{mm}$ hexagon bundle, individually connected to photodiodes. Primary data acquisition and display were undertaken with software written in LabVIEW (Texas Instruments). GFP alone (or its color mutants fused to optogenetic constructs to visually track expression levels) were usually virally transduced in cultured NRVM preparations. As such, conduction velocities were generally slower than observed in untransduced preparations, likely due to a previously reported effect of GFP itself to diminish velocities ${ }^{55}$. Conduction velocities may also have been slowed because we often enriched somewhat the usual fraction of cardiac fibroblasts in our NRVM cultures, to boost culture health and tolerance to strong expression of optogenetic constructs.

For di-4-ANEPPS (Molecular Probes) mapping, cells were stained with $10 \mu \mathrm{M}$ of dye for $7 \mathrm{~min}$. To record transmembrane potentials, the dye was excited perpendicular to the plane of the monolayer with an array of 26 high-power $530 \mathrm{~nm}$ LEDs (Kingbright) band-pass filtered at 530/50 nm. Fluorescence emission was long-pass filtered at $600 \mathrm{~nm}$ (PSCred; Brewer Science) before collection by the photodiode optic fiber array ${ }^{18}$.

For PGH1 mapping, cells were stained with $80 \mu \mathrm{M}$ of dye (from Guy Salama) for $9 \mathrm{~min}$. To record transmembrane potentials, the dye was excited perpendicular to the plane of the monolayer with an array of seven high-power $655 \mathrm{~nm}$ LEDs (Luxeon Star) band-pass filtered at 690/60 nm (Omega Optical). Fluorescence emission was long-pass filtered at $760 \mathrm{~nm}$ (Newport, catalog no. 20CGA-760) before collection by the photodiode fiber optic array. For simultaneous excitation of PGH1 and ChR2 or eNpHR3.0, the optical setup was modified for two orthogonal light paths using dichroic mirrors to split the red/blue or red/green lights at $475 \mathrm{~nm}$ (Omega Optical) or $618 \mathrm{~nm}$ (Omega Optical), respectively (Fig. 2a). ChR2 was excited with an array of seven high-power blue (447 nm) LEDs (Luxeon Star) band-pass filtered at 440/ $60 \mathrm{~nm}$ (Omega Optical). eNpHR3.0 was excited with an array of seven high-power green $(530 \mathrm{~nm}$ ) LEDs (Luxeon Star) band-pass filtered at 560/50 nm (Omega Optical). The optics setup was controlled using a custom-built electronic circuit programmed for various light pulse protocols using MPLAB (Microchip MPLAB IDE version 8.76.00.00)

When used, Bay K 8644 was diluted in Tyrodes to a final concentration of $1 \mu \mathrm{M}$ Bay K 8644 and continuously superfused over the monolayers during transmembrane potential recordings.

Whole-cell patch-clamping. HEK293 cells were transduced with ChR2 or eNpHR3.0 lentivirus and maintained as a stable cell line (non-monoclonal) for voltage-clamp recordings. Room temperature, whole-cell recordings were performed using an Axopatch 200A amplifier (Axon Instruments). P/8 voltage leak subtraction was used, with series resistances of 1-2 M $\Omega$ and $>70 \%$ compensation. Currents were lowpass filtered at $2 \mathrm{kHz}$ (4-pole Bessel) and sampled at $10 \mathrm{kHz}$. The bath contained (in $\mathrm{mM}$ ): $138 \mathrm{NaCl}, 4 \mathrm{KCl}, 2 \mathrm{CaCl}_{2}, 1 \mathrm{MgCl}_{2} \cdot 6 \mathrm{H} 2 \mathrm{O}, 0.33 \mathrm{NaH}_{2} \mathrm{PO} 4,10 \mathrm{HEPES}, 5$ glucose. The pipette solution contained (in $\mathrm{mM}$ ): $129 \mathrm{~K}$-gluconate, $10 \mathrm{HEPES}, 10 \mathrm{KCl}, 1 \mathrm{MgCl}_{2}, 0.5$ EGTA, $10 \mathrm{MgATP}^{6}$. An array of seven blue (447 nm) LEDs (Luxeon Star) was used to excite ChR2 at a maximum power of $7.2 \mathrm{~mW} / \mathrm{mm}^{2}$, and an array of seven green $(530 \mathrm{~nm})$ LEDs (Luxeon Star) was used to excite eNpHR3.0 at a maximum power of $1.2 \mathrm{~mW} / \mathrm{mm}^{2}$. A holding potential of $0 \mathrm{mV}$ and an 8-s repetition interval were used. Data analysis via custom MATLAB software (Mathworks, MA).

For cardiac APWs, optogenetic stimulatory light was turned on $500 \mathrm{~ms}$ before and after acquiring optogenetic currents during voltage-clamp with the APW. The APW utilized NRVM action potentials, as recorded using optical mapping with PGH1, and scaled as reported ${ }^{56}$.

Confocal imaging. NRVM monolayers bearing YFP-tagged ChR2 or eNpHR3.0 were imaged with a $60 \times$ water objective on an Olympus FV300 confocal microscope. An Argon-515 nm laser line was used for excitation, and emission fluorescence was filtered at $535 / 30 \mathrm{~nm}$.

Data analysis of optical signals. Custom MATLAB software (Mathworks, MA) was used for post-processing of optical signals. All signals were de-trended with a linear or polynomial fit and normalized to a range from 0 to 1 . To select activation and repolarization times, optical signals were filtered using a low-pass Butterworth filter at $35 \mathrm{~Hz}$. APD was calculated at $80 \%$ of repolarization $\left(\mathrm{APD}_{80}\right)$ (Fig. $5 \mathrm{c}$ ). Average action potentials were generated by aligning waveforms at $50 \%$ of depolarization. Activation maps for di-4-ANEPPS and PGH1 were constructed from activation times determined from the time of the stimulus to the instant at which waveforms first achieved $50 \%$ of peak. Colormaps represent activation times in ms. Activation maps for ChR2 triggered action potentials (Fig. 4a) were constructed from activation times, where time zero in the activation isochrone map corresponds to the start of the bluelight pulse. 
Gap compensation for blue- and green-light bleed-through. At times, multiple pulses of blue or green light were delivered during the plateau and repolarization phase of the action potentials. The green- or blue-light pulses caused a baseline shift in our optical signals (Supplementary Fig. S1), as the photodiode array was positioned directly in line with illuminating light, and commercially available 760-nm, long-pass, emission filters imperfectly excluded the strong incoming light pulses. Accordingly, these periods of baseline shift were excluded and manifest as 'gaps' in our optical records. To estimate the trajectory of voltage during these gaps produced by blue-light pulses (Fig. 5), we utilized a custom curve-fitting algorithm. First, each action potential was linearly de-trended and normalized to a range from 0 to 1 . Then, the control action potential (Fig. 5 (o)) was least-square-error fitted with a phenomenological function comprised of the weighted sum of three Hill equations:

$$
F_{\text {fit }}=A \cdot \frac{t^{n_{\text {hill 1 }}}}{t^{n_{\text {hill1 }}}+K_{\mathrm{d} 1}{ }^{n_{\text {hill1 }}}}+B \cdot \frac{t^{n_{\text {hill2 }}}}{t^{n_{\text {hill2 }}}+K_{\mathrm{d} 2}{ }^{n_{\text {hill2 }}}}+C \cdot \frac{t^{n_{\text {hill3 }}}}{t^{n_{\text {hill3 }}}+K_{\mathrm{d} 3} n_{\text {hill3 }}}
$$

Next, the elongation of APD due to a single blue pulse (Fig. $5 c$ (i)) was fitted by a biexponential function from the time of the start of the blue pulse (Eq. 1).

$$
F_{\text {fit_bluepulse }}=D \cdot\left[\exp \left(-\left(t-t_{\text {pulse }}\right) / \tau_{\text {slow }}\right)-\exp \left(-\left(t-t_{\text {pulse }}\right) / \tau_{\text {fast }}\right)\right]^{2}
$$

In particular, the difference of the action potential with and without the single blue pulse was least-square-error fitted with this bi-exponential function, and then this function (Eq. 2) was added to the control action-potential curve fit (Eq. 1). To obtain the curve fit of the two blue pulse action potential, the difference of the action potential with one and two blue pulses was fit with another bi-exponential function, and then this bi-exponential fit was added to the single blue pulse action potential curve fit (Fig. $5 c$ (ii)). The same process was successively performed for three and four blue pulse action potentials (Fig. $5 c$ (iii-iv)) until all fits were obtained (Fig. $5 c$, all curve fits on bottom right). In this manner, we minimized to a single gap the missing data to be estimated in fitting each pulse component (Eq. 2), thereby increasing precision. This 'gap compensation' procedure allowed for a continuous estimate of the voltage trajectory, despite the gaps apparent in optical voltage signals.

For gaps induced by green-light pulses, the lower power of these pulses enabled a simple offset to be added to signals during baseline shifts, so as to produce a corrected signal that was approximately continuous at pulse onsets and offsets (Figs. 4 and 6).

1. Boyden, E. S., Zhang, F., Bamberg, E., Nagel, G. \& Deisseroth, K. Millisecondtimescale, genetically targeted optical control of neural activity. Nat Neurosci 8 , 1263-1268 (2005).

2. Zhang, F. et al. Multimodal fast optical interrogation of neural circuitry. Nature 446, 633-639 (2007).

3. Nagel, G. et al. Channelrhodopsin-2, a directly light-gated cation-selective membrane channel. Proc Natl Acad Sci U S A 100, 13940-13945 (2003).

4. Oesterhelt, D. \& Stoeckenius, W. Rhodopsin-like protein from the purple membrane of Halobacterium halobium. Nat New Biol 233, 149-152 (1971).

5. Zhang, F., Wang, L. P., Boyden, E. S. \& Deisseroth, K. Channelrhodopsin-2 and optical control of excitable cells. Nat Methods 3, 785-792 (2006).

6. Gradinaru, V., Thompson, K. R. \& Deisseroth, K. eNpHR: a Natronomonas halorhodopsin enhanced for optogenetic applications. Brain Cell Biol 36, 129-139 (2008).

7. Hofmann, B. et al. Light induced stimulation and delay of cardiac activity. Lab Chip 10, 2588-2596 (2010).

8. Bruegmann, T. et al. Optogenetic control of heart muscle in vitro and in vivo. Nat Methods 7, 897-900 (2010).

9. Arrenberg, A. B., Stainier, D. Y., Baier, H. \& Huisken, J. Optogenetic control of cardiac function. Science 330, 971-974 (2010).

10. Abilez, O. J. et al. Multiscale computational models for optogenetic control of cardiac function. Biophys J 101, 1326-1334 (2011).

11. Abilez, O. J. Cardiac optogenetics. Conf Proc IEEE Eng Med Biol Soc 2012, 1386-1389, doi:10.1109/EMBC.2012.6346197 (2012).

12. Jia, Z. et al. Stimulating cardiac muscle by light: cardiac optogenetics by cell delivery. Circ Arrhythm Electrophysiol 4, 753-760 (2011).

13. Nussinovitch, U., Shinnawi, R. \& Gepstein, L. Modulation of Cardiac Tissue Electrophysiological Properties with Light-Sensitive Proteins. Cardiovasc Res, doi:10.1093/cvr/cvu037 (2014).

14. Kirkton, R. D. \& Bursac, N. Genetic engineering of somatic cells to study and improve cardiac function. Europace 14 Suppl 5, v40-v49, doi:10.1093/europace/ eus269 (2012)

15. Wong, J., Abilez, O. J. \& Kuhl, E. Computational Optogenetics: A Novel Continuum Framework for the Photoelectrochemistry of Living Systems. J Mech Phys Solids 60, 1158-1178, doi:10.1016/j.jmps.2012.02.004 (2012).

16. Boyle, P. M., Williams, J. C., Ambrosi, C. M., Entcheva, E. \& Trayanova, N. A. A comprehensive multiscale framework for simulating optogenetics in the heart. Nat Commun 4, 2370, doi:10.1038/ncomms3370 (2013).

17. Williams, J. C. et al. Computational optogenetics: empirically-derived voltageand light-sensitive channelrhodopsin-2 model. PLoS Comput Biol 9, e1003220, doi:10.1371/journal.pcbi.1003220 (2013).

18. Bian, W. \& Tung, L. Structure-related initiation of reentry by rapid pacing in monolayers of cardiac cells. Circ Res 98, e29-38 (2006).

19. Entcheva, E. Cardiac optogenetics. Am J Physiol Heart Circ Physiol 304, H1179-1191, doi:10.1152/ajpheart.00432.2012 (2013).
20. Antzelevitch, C. et al. Loss-of-function mutations in the cardiac calcium channel underlie a new clinical entity characterized by ST-segment elevation, short QT intervals, and sudden cardiac death. Circulation 115, 442-449 (2007)

21. Giudicessi, J. R. \& Ackerman, M. J. Potassium-channel mutations and cardiac arrhythmias-diagnosis and therapy. Nat Rev Cardiol 9, 319-332 (2012).

22. Wilde, A. A. \& Bezzina, C. R. Genetics of cardiac arrhythmias. Heart 91, 1352-1358 (2005).

23. Feldbauer, K. et al. Channelrhodopsin-2 is a leaky proton pump. Proc Natl Acad Sci U S A 106, 12317-12322 (2009).

24. Fromherz, P. \& Muller, C. O. Voltage-sensitive fluorescence of amphiphilic hemicyanine dyes in neuron membrane. Biochim Biophys Acta 1150, 111-122 (1993).

25. Salama, G. et al. Properties of new, long-wavelength, voltage-sensitive dyes in the heart. J Membr Biol 208, 125-140 (2005).

26. Patrick, M. J. et al. Enhanced aqueous solubility of long wavelength voltagesensitive dyes by covalent attachment of polyethylene glycol. Org Biomol Chem $\mathbf{5}$, 3347-3353 (2007).

27. Hess, P., Lansman, J. B. \& Tsien, R. W. Different modes of Ca channel gating behaviour favoured by dihydropyridine Ca agonists and antagonists. Nature 311, 538-544 (1984).

28. Weidmann, S. Effect of current flow on the membrane potential of cardiac muscle. J Physiol 115, 227-236 (1951).

29. Gaughan, J. P., Hefner, C. A. \& Houser, S. R. Electrophysiological properties of neonatal rat ventricular myocytes with alphal-adrenergic-induced hypertrophy. Am J Physiol 275, H577-590 (1998).

30. Kamiya, K., Guo, W., Yasui, K. \& Toyama, J. Hypoxia inhibits the changes in action potentials and ion channels during primary culture of neonatal rat ventricular myocytes. J Mol Cell Cardiol 31, 1591-1598, doi:10.1006/ jmcc.1999.0992 (1999).

31. Chow, B. Y. et al. High-performance genetically targetable optical neural silencing by light-driven proton pumps. Nature 463, 98-102 (2010).

32. Matiukas, A. et al. New near-infrared optical probes of cardiac electrical activity. Am J Physiol Heart Circ Physiol 290, H2633-2643 (2006).

33. Kralj, J. M., Douglass, A. D., Hochbaum, D. R., Maclaurin, D. \& Cohen, A. E. Optical recording of action potentials in mammalian neurons using a microbial rhodopsin. Nat Methods 9, 90-95 (2011).

34. Jin, L. et al. Single action potentials and subthreshold electrical events imaged in neurons with a fluorescent protein voltage probe. Neuron 75, 779-785 (2012).

35. St-Pierre, F. et al. High-fidelity optical reporting of neuronal electrical activity with an ultrafast fluorescent voltage sensor. Nat Neurosci 17, 884-889, doi:10.1038/nn.3709 (2014).

36. Fenno, L., Yizhar, O. \& Deisseroth, K. The development and application of optogenetics. Annu Rev Neurosci 34, 389-412 (2011).

37. Deo, M., Boyle, P. M., Kim, A. M. \& Vigmond, E. J. Arrhythmogenesis by single ectopic beats originating in the Purkinje system. Am J Physiol Heart Circ Physiol 299, H1002-1011 (2010).

38. McDowell, K. S., Arevalo, H. J., Maleckar, M. M. \& Trayanova, N. A. Susceptibility to arrhythmia in the infarcted heart depends on myofibroblast density. Biophys J 101, 1307-1315 (2011)

39. Trayanova, N. A. Whole-heart modeling: applications to cardiac electrophysiology and electromechanics. Circ Res 108, 113-128 (2011).

40. Choi, G. B. et al. Driving opposing behaviors with ensembles of piriform neurons. Cell 146, 1004-1015, doi:10.1016/j.cell.2011.07.041 (2011).

41. Weiss, J. N., Garfinkel, A., Karagueuzian, H. S., Chen, P. S. \& Qu, Z. Early afterdepolarizations and cardiac arrhythmias. Heart Rhythm 7, 1891-1899, doi:10.1016/j.hrthm.2010.09.017 (2010).

42. Wolff, M. R. et al. Alterations in left ventricular mechanics, energetics, and contractile reserve in experimental heart failure. Circ Res 70, 516-529 (1992).

43. Cho, H., Barth, A. S. \& Tomaselli, G. F. Basic science of cardiac resynchronization therapy: molecular and electrophysiological mechanisms. Circ Arrhythm Electrophysiol 5, 594-603 (2012).

44. Yazawa, M. et al. Using induced pluripotent stem cells to investigate cardiac phenotypes in Timothy syndrome. Nature 471, 230-234 (2011).

45. Dominguez-Rodriguez, A., Ruiz-Hurtado, G., Benitah, J. P. \& Gomez, A. M. The other side of cardiac $\mathrm{Ca}(2+)$ signaling: transcriptional control. Front Physiol 3, 452 (2012).

46. Kehat, I. \& Molkentin, J. D. Molecular pathways underlying cardiac remodeling during pathophysiological stimulation. Circulation 122, 2727-2735 (2010).

47. Nattel, S., Maguy, A., Le Bouter, S. \& Yeh, Y. H. Arrhythmogenic ion-channel remodeling in the heart: heart failure, myocardial infarction, and atrial fibrillation. Physiol Rev 87, 425-456 (2007).

48. Wang, Y. \& Hill, J. A. Electrophysiological remodeling in heart failure. J Mol Cell Cardiol 48, 619-632 (2010).

49. Yu, J. et al. Activation of beta(3)-adrenoceptor promotes rapid pacing-induced atrial electrical remodeling in rabbits. Cell Physiol Biochem 28, 87-96 (2011)

50. Baartscheer, A. et al. Chronic inhibition of $\mathrm{Na}+/ \mathrm{H}+$-exchanger attenuates cardiac hypertrophy and prevents cellular remodeling in heart failure. Cardiovasc Res 65, 83-92 (2005).

51. Boyle, P. M., Entcheva, E. \& Trayanova, N. A. See the light: can optogenetics restore healthy heartbeats? And, if it can, is it really worth the effort? Expert Rev Cardiovasc Ther 12, 17-20, doi:10.1586/14779072.2014.864951 (2014). 
52. Bolli, R. et al. Cardiac stem cells in patients with ischaemic cardiomyopathy (SCIPIO): initial results of a randomised phase 1 trial. Lancet 378, 1847-1857 (2011).

53. Johnston, P. V. et al. Engraftment, differentiation, and functional benefits of autologous cardiosphere-derived cells in porcine ischemic cardiomyopathy. Circulation 120, 1075-1083 (2009).

54. Zhang, F. et al. Optogenetic interrogation of neural circuits: technology for probing mammalian brain structures. Nat Protoc 5, 439-456 (2010).

55. Sekar, R. B. et al. Lentiviral vector-mediated expression of GFP or Kir2.1 alters the electrophysiology of neonatal rat ventricular myocytes without inducing cytotoxicity. Am J Physiol Heart Circ Physiol 293, H2757-2770, doi:10.1152/ ajpheart.00477.2007 (2007).

56. Fermini, B. \& Schanne, O. F. Determinants of action potential duration in neonatal rat ventricle cells. Cardiovasc Res 25, 235-243 (1991).

\section{Acknowledgments}

The authors thank M. Ben-Johny for help with software development, W. Yang for preparing cells, R. Sekar for lentivirus production and NRVM isolation protocols, S. Thompson with NRVM isolation optimization, S. Weinberg and R. Joshi-Mukherjee with optical mapping assistance, and $\mathrm{H}$. Fatemi-Shariatpanahi with the initial opto-electronics development. This work was funded by the National Institutes of Health (NIH) MERIT award 5R37HL076795 (DTY).

\section{Author contributions}

S.A.P. contributed to all aspects of the experiments, coded custom MATLAB scripts, and analyzed data. S.R.L. contributed importantly to electronics and optical design of the apparatus, acquired the confocal images, and helped to analyze and prepare the figures. S.A.P. and S.R.L. performed patch-clamp experiments. S.A.P., S.R.L., L.T. and D.T.Y. designed and constructed the optics setup. D.T.Y. devised the gap compensation method, supervised the work, and helped conceive the project. D.T.Y. and S.A.P. wrote the manuscript, with input from all other authors.

\section{Additional information}

Supplementary information accompanies this paper at http://www.nature.com/ scientificreports

Competing financial interests: The authors declare no competing financial interests.

How to cite this article: Park, S.A., Lee, S.-R., Tung, L. \& Yue, D.T. Optical mapping of optogenetically shaped cardiac action potentials. Sci. Rep. 4, 6125; DOI:10.1038/srep06125 (2014).

(c) (i) (2) This work is licensed under a Creative Commons Attribution-NonCommercialShareAlike 4.0 International License. The images or other third party material in this article are included in the article's Creative Commons license, unless indicated otherwise in the credit line; if the material is not included under the Creative Commons license, users will need to obtain permission from the license holder in order to reproduce the material. To view a copy of this license, visit http:// creativecommons.org/licenses/by-nc-sa/4.0/ 